\title{
A Feasibility Study of Personalized Prescription Schemes for Glioblastoma Patients Using a Proliferation and Invasion Glioma Model
}

\author{
Minsun Kim ${ }^{1, *}$, Jakob Kotas ${ }^{2}$, Jason Rockhill ${ }^{1}$ and Mark Phillips ${ }^{1}$ \\ 1 Department of Radiation Oncology, University of Washington, Seattle, WA 98195, USA; \\ jkrock@uw.edu (J.R.); markp@uw.edu (M.P.) \\ 2 Department of Mathematics, University of Portland, Portland, OR 97203, USA; jkotas@uw.edu \\ * Correspondence: mk688@uw.edu; Tel.: +1-206-598-8133
}

Academic Editor: David Wong

Received: 6 April 2017; Accepted: 11 May 2017; Published: 13 May 2017

\begin{abstract}
Purpose: This study investigates the feasibility of personalizing radiotherapy prescription schemes (treatment margins and fractional doses) for glioblastoma (GBM) patients and their potential benefits using a proliferation and invasion (PI) glioma model on phantoms. Methods and Materials: We propose a strategy to personalize radiotherapy prescription schemes by simulating the proliferation and invasion of the tumor in 2D according to the PI glioma model. We demonstrate the strategy and its potential benefits by presenting virtual cases, where the standard and personalized prescriptions were applied to the tumor. Standard prescription was assumed to deliver 46 Gy in 23 fractions to the initial, gross tumor volume $\left(G V_{1}\right)$ plus a $2 \mathrm{~cm}$ margin and an additional $14 \mathrm{~Gy}$ in 7 fractions to the boost $\mathrm{GTV}_{2}$ plus a $2 \mathrm{~cm}$ margin. The virtual cases include the tumors with a moving velocity of 0.029 (slow-move), 0.079 (average-move), and 0.13 (fast-move) $\mathrm{mm} /$ day for the gross tumor volume (GTV) with a radius of 1 (small) and 2 (large) $\mathrm{cm}$. For each tumor size and velocity, the margin around $\mathrm{GTV}_{1}$ and $\mathrm{GTV}_{2}$ was varied between $0-6 \mathrm{~cm}$ and $1-3 \mathrm{~cm}$, respectively. Equivalent uniform dose (EUD) to normal brain was constrained to the EUD value obtained by using the standard prescription. Various linear dose policies, where the fractional dose is linearly decreasing, constant, or increasing, were investigated to estimate the temporal effect of the radiation dose on tumor cell-kills. The goal was to find the combination of margins for GTV 1 and $G_{T V}$ and a linear dose policy, which minimize the tumor cell-surviving fraction (SF) under a normal tissue constraint. The efficacy of a personalized prescription was evaluated by tumor EUD and the estimated survival time. Results: The personalized prescription for the slow-move tumors was to use 3.0-3.5 cm margins for $\mathrm{GTV}_{1}$, and a $1.5 \mathrm{~cm}$ margin for $\mathrm{GTV}_{2}$. For the average- and fast-move tumors, it was optimal to use a $6.0 \mathrm{~cm}$ margin for $\mathrm{GTV}_{1}$ and then 1.5-3.0 $\mathrm{cm}$ margins for $\mathrm{GTV}_{2}$, suggesting a course of whole brain therapy followed by a boost to a smaller volume. It was more effective to deliver the boost sequentially using a linearly decreasing fractional dose for all tumors. Personalized prescriptions led to surviving fractions of $0.001-0.465 \%$ compared to the standard prescription, and increased the tumor EUDs by $25.3-49.3 \%$ and estimated survival times by 7.6-22.2 months. Conclusions: Personalizing treatment margins based on the measured proliferative capacity of GBM tumor cells can potentially lead to significant improvements in tumor cell kill and related clinical outcomes.
\end{abstract}

Keywords: radiotherapy treatment planning; glioblastoma; mathematical model

\section{Introduction}

Glioblastoma is highly heterogeneous, where different cells within the tumor respond to different types of therapy, leading to a treatment regimen involving multiple modalities [1]. Despite aggressive 
treatment with surgical resection and chemo-radiation, the prognosis remains poor with short median survival of less than 15 months with radiotherapy plus concomitant and adjuvant Temozolomide [2], and there has not been a dramatic improvement in survival over the last several decades [3].

Studies show that the extent of the surgical resection is an important prognostic factor in Glioblastoma Multiforme (GBM) [4]. However, most patients, including those who received the most radical surgical excisions, die of recurrent tumors [5]. Ogura et al. analyzed the locations of recurrent tumors for 21 patients [6]. Their study showed that initial recurrence occurred centrally at first (67\% of the total patients) but distant recurrences were also observed in $19 \%$ of the patients. Cumulative recurrence patterns showed distant recurrences in the majority of the patients (88\%). These clinical observations suggest that tumor cells spread throughout the brain. Swanson et al. published a mathematical model to describe the spatio-temporal dynamics of glioma cells using a reaction-diffusion equation [7]. Their model uses patient-specific, net rates of proliferation and invasion obtained from serial Magnetic Resonance Imaging (MRIs) as input parameters to describe how quickly the tumor grows and migrates in the spatiotemporal space. Therefore, their model predicts the tumor (both gross and microscopic disease) growth beyond what can be seen with current imaging technology. The prediction of tumor growth using their model also agrees with the clinical observation that glioma cells invade into normal brain. Therefore, the optimal extent of the treatment volume may affect clinical outcomes.

The standard treatment includes maximal safe resection followed by concurrent chemotherapy and radiotherapy to the resection cavity with a margin to eradicate the remaining tumor cells [8]. Radiation therapy of $60 \mathrm{~Gy}$ in 30 fractions is given either to one tumor volume or in two phases, where $46 \mathrm{~Gy}$ is given to a large volume and an additional $14 \mathrm{~Gy}$ to a reduced volume. The initial target volume consists of the gross tumor volume $\left(\mathrm{GTV}_{1}\right)$, which is defined by either the T2 or Fluid-Attenuated Inversion Recovery (FLAIR) signal on the post-operative MRI scan, plus a margin. The boost target volume $\left(\mathrm{GTV}_{2}\right)$ is based on the contrast-enhanced, T1 MRI scan, plus a margin [6]. A variable margin around $\mathrm{GTV}_{1}$ and $\mathrm{GTV}_{2}$ is currently used to define the clinical target volumes for each phase reflecting the difficulty in determining the exact extent of the tumor cells. Ghose et al. surveyed the variability in the practice patterns of Canadian radiation oncologists treating GBM [9]. They reported a significant variation in the margins used, ranging from $0.5 \mathrm{~cm}$ to $3.0 \mathrm{~cm}$, with some of them using more than one standard margin. Multiple studies indicate that there is a lack of consensus in the most appropriate target volume to be treated [9-11].

The purpose of this study is to investigate the feasibility of personalizing prescription schemes using a tumor growth model, which includes patient-specific parameters, and to estimate their potential benefits on clinical outcome. Specifically, we assume that the spatiotemporal dynamics of the tumor follow the proliferation and invasion (PI) glioma model described in [12]. In this study, the prescription

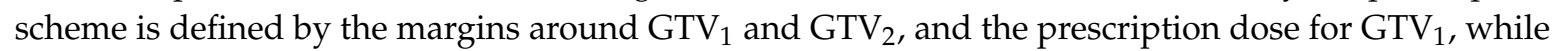
keeping the total dose for $\mathrm{GTV}_{2}$ to $60 \mathrm{~Gy}$. We also investigated the temporal effect of the radiation on the tumor damage by linearly increasing or decreasing a fractional dose, and delivering a boost phase either concurrently or sequentially. The goal is to find the most effective treatment margins and temporal dose policy in killing tumor-cells without increasing the generalized equivalent uniform dose (gEUD) to normal brain compared to the current standard-of-care. The efficacy of the personalized prescription schemes was evaluated by comparing the cell-surviving fraction (SF), tumor EUD, and the estimated survival time with those of the standard-of-care.

\section{Methods and Materials}

\subsection{Prescription Geometry}

A standard prescription to treat GBM was assumed to deliver a total dose of $60 \mathrm{~Gy}\left(=\mathrm{TD}_{2}\right)$ in two phases, with $46 \mathrm{~Gy}\left(=\mathrm{TD}_{1}\right)$ being delivered in 23 fractions in the initial phase to $\mathrm{GTV}_{1}$ plus a $2 \mathrm{~cm}$ margin followed by a boost of $14 \mathrm{~Gy}$ in 7 fractions to $\mathrm{GTV}_{2}$ plus a $2 \mathrm{~cm}$ margin (Figure 1) [11]. 


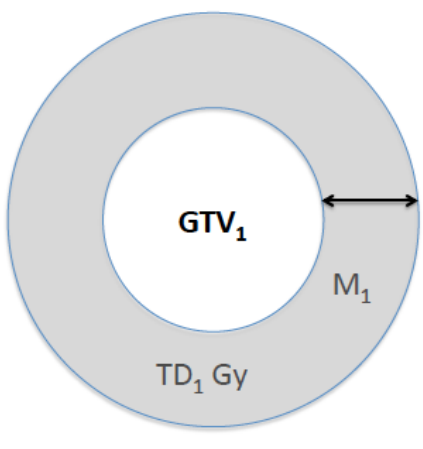

(a) Initial phase

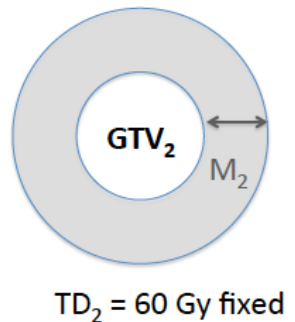

(b) Boost phase

Figure 1. Prescription geometry. For the standard prescription, $\mathrm{TD}_{1}=46 \mathrm{~Gy}, \mathrm{TD}_{2}=60 \mathrm{~Gy}$, and $\mathrm{M}_{1}=\mathrm{M}_{2}=2 \mathrm{~cm}$.

\subsection{Tumor Growth Simulation Using a PI Glioma Model}

We used the spatio-temporal model of the tumor proliferation and invasion under the effect of radiotherapy as presented in [12]. Let $D$ be a diffusion coefficient, and $s$ be the length of a radiotherapy session. Let $\rho$ and $k$ be the parameters related to the rate of cell proliferation and the carrying capacity of the tissue, respectively. Then the tumor dynamics under radiotherapy can be modeled as follows:

$$
\frac{\partial c}{\partial t}=\underbrace{\nabla(D \nabla c)}_{\text {Net dispersion }}+\underbrace{\rho c(1-c / k)}_{\text {Net proliferation }}-\underbrace{R c / s}_{\text {Net cell-kills }}
$$

where $c=c(x, t)$ is the glioma cell concentration and $R=R(x, t)$ is the cell loss due to radiotherapy at location $x$ and time $t$. Equation (1) describes the rate of change in the glioma cell concentration at any given point in the brain in terms of the tumor dispersion velocity and proliferation rate, which vary among different patients and are measurable from two or more MRIs taken at different times. The linear quadratic (LQ) cell-survival model [13] gives the loss of glioma cells during radiotherapy as follows:

$$
R(x, t)=\left\{\begin{array}{c}
1-\exp \left[-\alpha\left(d_{t}(\boldsymbol{x})+\frac{d_{t}(\boldsymbol{x})^{2}}{\alpha / \beta}\right)\right]: \text { during radiotherapy } \\
0 \text { : between fractions, }
\end{array}\right.
$$

where $d_{t}(x)$ is the dose administered at time $t$ to location $x$, and $\alpha$ and $\beta$ are tissue specific radio-sensitivity parameters. We used the two-dimensional finite difference method to approximately solve for $c(x, y ; t)$ in Equation (1).

The tumor volume was simulated using Equation (1) with the following parameters as in [3]: $\rho=0.012 /$ day, $\alpha=0.035 \mathrm{~Gy}, \alpha / \beta=10 \mathrm{~Gy}$, and $k=10^{9} / \mathrm{cm}^{3}$. Swanson et al. reported in [14] that the measured values of $D$ from serial MRIs for 70 patients ranged from 0.24 to $35.92 \mathrm{~mm}^{2} /$ day (mean 10.52 , median $9.83 \mathrm{~mm}^{2} /$ day) and used Fisher's approximation to calculate an approximate radial velocity of expansion of the edge of a tumor $(v=\sqrt{2 \rho D})$. The velocity $(v)$ ranged between 0.0118 and $0.1438 \mathrm{~mm} /$ day. Based on this report, we used three values of $D=0.017,0.13$, and $0.34 \mathrm{~mm} /$ day to characterize slow-, average-, and fast-move tumors.

Swanson et al. hypothesized that the circumference of the T2 MR hyper-intense area $\left(G_{T V}\right)$ and T1-Gd MR hyper-intense area $\left(\mathrm{GTV}_{2}\right)$ represent $16 \%$ and $80 \%$ of the maximum tumor cell concentration [15], respectively. We studied two different $\mathrm{GTV}_{2}$ sizes: $\mathrm{GTV}_{1}$ was obtained when the radius of $\mathrm{GTV}_{2}$ reached 1.0 and $2.0 \mathrm{~cm}$ from the initial condition of $\mathrm{c}_{0}\left(\mathrm{x}_{0}, \mathrm{y}_{0} ; 0\right)=\mathrm{L}^{3} \mathrm{e}^{-100\left(\mathrm{x}_{0}^{2}+\mathrm{y}_{0}{ }_{0}\right)}$, where $\mathrm{L}$ is the length of the computational domain [12]. The corresponding radius of $\mathrm{GTV}_{1}$ was recorded for each $\mathrm{GTV}_{2}$ (Table 1). 
Table 1. Tumor size and moving velocity used in this study.

\begin{tabular}{ccc}
\hline Moving Velocity (mm/day) & GTV $_{\mathbf{1}}$ Radius $(\mathbf{c m})$ & GTV $_{\mathbf{2}}$ Radius $(\mathbf{c m})$ \\
\hline \multirow{2}{0}{$\begin{array}{c}0.029 \\
\text { (slow-move) }\end{array}$} & 1.7 & 1.0 (small) \\
\hline $\begin{array}{c}0.079 \\
\text { (average-move) }\end{array}$ & 2.7 & 2.0 (large) \\
\cline { 2 - 3 } $\begin{array}{c}0.13 \\
\text { (fast-move) }\end{array}$ & 2.7 & 1.0 (small) \\
\hline \cline { 2 - 3 } & 4.2 & 2.0 (large) \\
\hline
\end{tabular}

Gross tumor volume $\left(\mathrm{GTV}_{1}\right)$ was simulated using the parameters of proliferation rate $\left(\rho=0.012\right.$ day $\left.^{-1}\right)$ and carrying capacity $\left(k=10^{9} \mathrm{~cm}^{-3}\right)$ for each size of $\mathrm{GTV}_{2}$.

We simulated the tumor with and without modeling the resection cavity. The resection cavity was modeled by setting the number of tumor cells inside $\mathrm{GTV}_{2}$ to zero.

\subsection{Prescription Scheme Variables}

The goal is to find the treatment margins around $\mathrm{GTV}_{1}$ and $\mathrm{GTV}_{2}$ (i.e., $\mathrm{M}_{1}$ and $\mathrm{M}_{2}$ ) as well as $\mathrm{TD}_{1}$ delivered to $\mathrm{GTV}_{1}+\mathrm{M}_{1}$, which leads to the minimum total number of tumor cells after 30 fractions. The margins $\mathrm{M}_{1}$ and $\mathrm{M}_{2}$ were chosen from the sets $\{0,0.5,1.0, \cdots, 6.0\}$ and $\{1.0,1.5,2.0$, $2.5,3.0\}$, respectively. In our simulation, $\mathrm{TD}_{2}$ was fixed at $60 \mathrm{~Gy} . \mathrm{TD}_{1}$ was constrained such that the generalized equivalent uniform dose (gEUD) of normal brain equals the gEUD resulting from the standard prescription. This means that the treatment with a larger margin is feasible only with a smaller dose to ensure that the toxicity of the non-standard prescription scheme on the normal brain does not exceed the toxicity from the standard prescription. Normal brain gEUD was calculated as follows [16]:

$$
\begin{gathered}
\mathrm{gEUD}=\left(f_{1} \mathrm{TD}_{1}{ }^{a}+f_{2} \mathrm{TD}_{2}{ }^{a}\right)^{-a}, \\
f_{1}+f_{2} \leq 1, \mathrm{TD}_{2}=60 \mathrm{~Gy},
\end{gathered}
$$

where $f_{1}$ and $f_{2}$ are the fractional areas irradiated to $\mathrm{TD}_{1}$ and $\mathrm{TD}_{2}$, respectively, and $a$ is a tissue-specific sensitivity parameter. The sum of $f_{1}$ and $f_{2}$ would be less than 1 only if the irradiated area of the standard prescription is larger than the prescription scheme investigated, i.e., there is a fractional area $\left(1-f_{1}-f_{2}\right)$ with zero dose. TD 1 is obtained by constraining gEUD of the normal brain to be the same as the standard prescription:

$$
\left(f_{1}{ }^{\text {standard }} 46^{a}+f_{2} \text { standard } 6^{0 a}\right)^{-a}=\left(f_{1}{ }^{\text {non-standard }} \mathrm{TD}_{1}{ }^{a}+f_{2}{ }^{\text {non-standard }} 60^{a}\right)^{-a}
$$

Therefore, $\mathrm{TD}_{1}$ is a function of $\mathrm{M}_{1}, \mathrm{M}_{2}$, and the radii of $\mathrm{GTV}_{1}$ and $\mathrm{GTV}_{2}$. In this study, we used $a=5$ for normal brain $[15,17]$. We also varied $a$ between 3 and 7 to investigate the sensitivity of the optimal solutions on $a$. To examine the temporal effect of the radiation dose, five different, linear dose policies $\left(\mathrm{P}_{1}-\mathrm{P}_{5}\right)$ have been implemented as shown in Figure 2. The sum of the fractional doses is fixed at 60 Gy. $P_{3}$ represents the current, constant dose policy, in which the fractional dose is fixed at $2 \mathrm{~Gy}$. Linearly increasing (P1/P2) or decreasing dose (P4/P5) policies were implemented with an initial dose of 1.0/1.5 Gy for P1/P2 and 2.5/3.0 Gy for P4/P5 (Figure 2). We ignored the fractionation effects on the normal brain because the linear dose policies investigated in this study result in a clinically insignificant difference in the biologically effective dose (BED) using $\alpha / \beta=3$ Gy, i.e., 100, 100.9, and 103.4 Gy for P3, P2/P4, and P1/P5, respectively. 


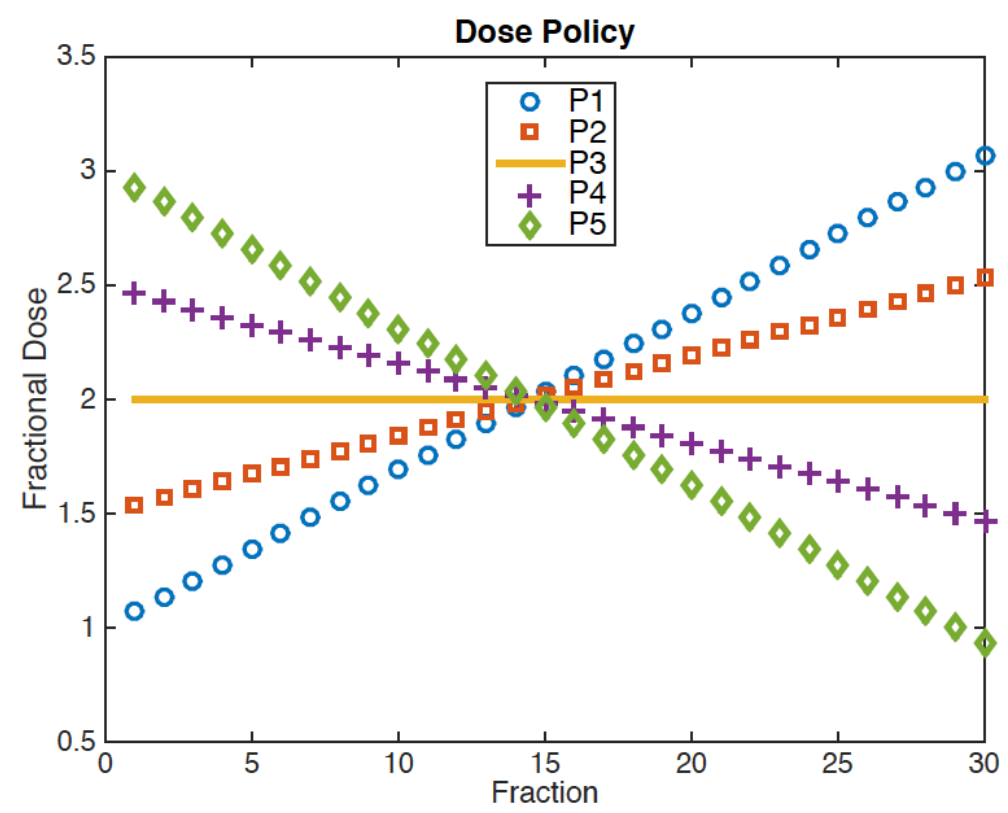

Figure 2. Non-stationary dose policies for the total dose of $60 \mathrm{~Gy}$ to $\mathrm{GTV}_{2}+\mathrm{M}_{2}: \mathrm{P}_{1} / \mathrm{P}_{2}$ and $\mathrm{P}_{4} / \mathrm{P}_{5}$ have a linearly increasing/decreasing fractional dose. $\mathrm{P}_{3}$ represents the constant dose per fraction as in the standard prescription.

A boost phase was delivered (i) sequentially or (ii) concurrently. In the sequential boost, $\mathrm{TD}_{1}$ was delivered to $G T V_{1}+M_{1}$ first with a fractional dose as shown in Figure 2, and then $\mathrm{TD}_{2}-\mathrm{TD}_{1}$ was additionally delivered to $\mathrm{GTV}_{2}+\mathrm{M}_{2}$ only. Therefore, the number of fractions in the initial phase varied depending on $\mathrm{TD}_{1}$ and the dose policy used. In the concurrent boost, $\mathrm{TD}_{1}$ and $\mathrm{TD}_{2}$ were simultaneously delivered in 30 fractions to $\mathrm{GTV}_{1}+\mathrm{M}_{1}$ and $\mathrm{GTV}_{2}+\mathrm{M}_{2}$, respectively.

\subsection{Evaluation Criteria}

The efficacy of each non-standard prescription scheme was evaluated by comparing the tumor cell-surviving fraction (SF) after 30 fractions relative to the SF that resulted from the standard prescription. In addition, the tumor EUD of the personalized prescription relative to the EUD from the standard prescription was calculated. The higher the EUD is, the higher the tumor cell-kill that is achieved. Let $\mathrm{SF}^{*}$ and $\mathrm{SF}^{0}$ be the tumor cell-surviving fraction after the treatment course using the personalized prescription and the standard prescription, respectively. Similarly, let EUD ${ }^{*}$ and EUD ${ }^{0}$ be the EUD from the personalized and the standard prescription, respectively. Then the surviving fraction can be written as:

$$
\begin{aligned}
& S F^{*}=\exp \left[-\alpha E U D^{*}-\beta\left(E U D^{*}\right)^{2} / N\right], \\
& S F^{0}=\exp \left[-\alpha E U D^{0}-\beta\left(E U D^{0}\right)^{2} / N\right],
\end{aligned}
$$

where $N=30$ fractions. Therefore, $\mathrm{EUD}^{*}$ as a function of $\mathrm{EUD}^{0}$ is given by:

$$
E U D^{*}=\left(\frac{N}{2 \beta}\right)\left(-\alpha+\sqrt{\alpha^{2}+\frac{4 \beta}{N}}\left(\alpha E U D^{0}+\frac{\beta\left(E U D^{0}\right)^{2}}{N}-\ln \left(\frac{S F^{*}}{S F^{0}}\right)\right)\right) .
$$

EUD $^{0}$ was calculated using Equation (3) with $a=-10$ for the tumor [17].

We also estimated the survival times for the standard and personalized prescriptions. Swanson et al. showed that their PI glioma model predicts the actual survival time by simulating the tumor growth until it reaches its fatal radius of $3 \mathrm{~cm}$ [18], which they used as an indicator of the interval to 
death. We let the tumor grow after the course of radiotherapy and measured the time required for $\mathrm{GTV}_{2}$ to reach a radius of $3 \mathrm{~cm}$ for the standard and personalized prescription schemes, respectively.

\section{Results}

Personalized prescription schemes were not affected by the following parameters: (i) modeling resection cavity in the tumor growth simulation (ii) radiobiological parameters $(\alpha / \beta)$ used in the LQ cell-survival model, and (iii) the EUD parameter, $a$. Therefore, all the results are presented in this section using $a=5$ for the normal brain and $\alpha / \beta=10 \mathrm{~Gy}$ for the tumor without the modeling resection cavity. In summary, Table 2 shows the personalized prescription scheme for each tumor size and velocity studied, which leads to the maximum cell-kills in the simulation. Table 3 shows the tumor EUD and the expected survival that results from using these personalized prescription schemes in Table 2. The percentage improvements are relative to the results from using the standard prescription.

\subsection{Sequential and Concurrent Boost}

Tumor SF for the personalized prescription was normalized to that for the standard prescription using:

$$
\text { Personalized } \mathrm{SF}^{*}(\%)=\frac{\mathrm{SF} \text { using personalized } \mathrm{M}_{1}, \mathrm{M}_{2} \text {, and } \mathrm{TD}_{1}}{\mathrm{SF} \text { using the standard prescription }} \times 100 \text {. }
$$

Personalized SF (P-SF) using the sequential boost was 18.3-61.3\% lower than the P-SF using the concurrent boost for all cases except for the fast-move, large tumor. In this case, the concurrent boost P-SF was $41.4 \%$ lower than the sequential boost P-SF.

For the sequential boost, the personalized $M_{1}$ ranged from $3.0-6.0 \mathrm{~cm}$, and $\mathrm{M}_{2}$ was $1.5 \mathrm{~cm}$ for all tumors except for the fast-move, large tumor. The corresponding $\mathrm{TD}_{1}$ ranged between 20.7 and 45.6 Gy. A linearly decreasing dose policy $\mathrm{P}_{5}$, i.e., a larger fraction size upfront, led to the smallest $\mathrm{SF}$ for all tumor sizes and velocities. P-SF for all tumors studied was $0.001-0.465 \%$ of the SF that resulted from the standard prescription (Table 2).

\subsection{Stationary Fractional Dose Policy}

To evaluate if the constant fractional dose policy $\left(\mathrm{P}_{3}\right)$ is clinically equivalent to the personalized dose policy, we compared SF using $\mathrm{P}_{3}$ to the standard prescription and to $\mathrm{P}_{5}$ (with $\mathrm{M}_{1}, \mathrm{M}_{2}$, and $\mathrm{TD}_{1}$ being fixed to their personalized values for both $\mathrm{P}_{3}$ and $\mathrm{P}_{5}$ ). For the different tumor characteristics (sizes and velocities), the $\mathrm{SF}$ using $\mathrm{P}_{3}$ relative to the $\mathrm{SF}$ using the standard prescription varied from $0.001-0.491 \%$ (sequential boost) and $0.002-0.330 \%$ (concurrent boost). Relative to P-SF, the stationary dose policy $\left(\mathrm{P}_{3}\right)$ resulted in a higher SF: 105.7-218.8\% (sequential) and 100.4-136.9\% (concurrent).

\subsection{Comparison of EUD and Estimated Survival Time}

We computed EUD of the tumor using the personalized prescription (Table 2) relative to that with the standard prescription. (Note that EUD $>100 \%$ indicates superiority of the personalized prescription; this is the opposite of the P-SF.) The EUD using the personalized prescription was $123.8-149.3 \%$ and $125.3-146.5 \%$ of the EUD from the standard prescription for the sequential and concurrent boost, respectively. The EUD using $\mathrm{P}_{3}$ and personalized $\mathrm{M}_{1}, \mathrm{M}_{2}$, and $\mathrm{TD}_{1}$ was $123.6-147.5 \%$ and $125.3-146.1 \%$ of the EUD resulting from the standard prescription for the sequential and concurrent boost, respectively. Therefore, the difference in the tumor EUD between $\mathrm{P}_{5}$ and $\mathrm{P}_{3}$ with a sequential boost is less than $3 \%$ for all tumors if $\mathrm{M}_{1}, \mathrm{M}_{2}$, and $\mathrm{TD}_{1}$ are fixed at their personalized values.

To calculate the effects of radiation therapy on the subsequent growth of the tumor, and hence the length of survival according to the model described in [7], the tumor cell distribution at the end of 30 fractions for each specific prescription was used as the starting point for calculating post-therapy tumor growth and spread. Then tumor growth was calculated according to Equation (1) with $R=0$ (no radiation), and the time in months it took for the tumor to reach its fatal radius of $3.0 \mathrm{~cm}$ was 
determined. For the post-radiotherapy tumor growth, serial MRIs were not available to measure the patient-specific $\rho / D$. Therefore, we assumed that $\rho$ was increased to $0.020 /$ day to calibrate the survival time for the average-move tumors treated with the standard prescription to be approximately 19 months [19]. With this assumption, the optimal prescription led to a 7.6-22.2 months longer survival time than the standard prescription (Table 3).

Table 2. Personalized prescription schemes.

\begin{tabular}{|c|c|c|c|c|c|c|c|}
\hline Velocity (mm/day) & $\begin{array}{l}\text { GTV2 Radius } \\
\text { (cm) }\end{array}$ & P-SF (\%) & $\mathrm{P}-\mathrm{M}_{1}(\mathrm{~cm})$ & $\begin{array}{c}\text { P-TD }_{1} \\
\text { (Gy) }\end{array}$ & $\mathrm{P}-\mathrm{M}_{2}(\mathrm{~cm})$ & $\begin{array}{l}\text { P-Boost } \\
\text { Delivery }\end{array}$ & $\begin{array}{l}\text { P-Dose } \\
\text { Policy }\end{array}$ \\
\hline \multirow{2}{*}{$\begin{array}{c}0.029 \\
\text { (slow-move) }\end{array}$} & 1.0 & 0.021 & 3.0 & 45.6 & 1.5 & Sequential & $\mathrm{P}_{5}$ \\
\hline & 2.0 & 0.048 & 3.5 & 43.7 & 1.5 & Sequential & $\mathrm{P}_{5}$ \\
\hline \multirow{2}{*}{$\begin{array}{c}0.079 \\
\text { (average-move) }\end{array}$} & 1.0 & 0.001 & $6.0(\max )$ & 37.0 & 1.5 & Sequential & $\mathrm{P}_{5}$ \\
\hline & 2.0 & 0.001 & $6.0(\max )$ & 38.3 & 1.5 & Sequential & $\mathrm{P}_{5}$ \\
\hline \multirow{2}{*}{$\begin{array}{c}0.13 \\
\text { (fast-move) }\end{array}$} & 1.0 & 0.001 & $6.0(\max )$ & 37.0 & 1.5 & Sequential & $P_{5}$ \\
\hline & 2.0 & 0.328 & $6.0(\max )$ & 32.9 & 2.5 & Concurrent & $\mathrm{P}_{5}$ \\
\hline
\end{tabular}

Personalized (P-) treatment margins $\left(\mathrm{M}_{1}\right.$ for $\mathrm{GTV}_{1}$ and $\mathrm{M}_{2}$ for $\left.\mathrm{GTV}_{2}\right)$, total dose in the initial phase $\left(\mathrm{TD}_{1}\right)$, and dose policy are shown for each tumor studied. The tumor cell-surviving fraction (SF) using the personalized prescription is shown as a percentage of SF obtained from the standard prescription $\left(M_{1}=M_{2}=2 \mathrm{~cm}\right.$, $\left.\mathrm{TD}_{1}=46 \mathrm{~Gy}\right)$.

Table 3. Efficacy of personalized prescriptions.

\begin{tabular}{ccccc}
\hline Velocity (mm/day) & $\begin{array}{c}\text { GTV2 } \\
\text { Radius (cm) }\end{array}$ & $\begin{array}{c}\text { P-Tumor } \\
\text { EUD (\%) }\end{array}$ & $\begin{array}{c}\text { Std. Estimated Survival } \\
\text { Time (Months) }\end{array}$ & $\begin{array}{c}\text { P-Estimated Survival } \\
\text { Time (Months) }\end{array}$ \\
\hline $\begin{array}{c}0.029 \\
\text { (slow-move) }\end{array}$ & 1.0 & 134.3 & 53.5 & 62.5 \\
\hline \multirow{2}{*}{$\begin{array}{c}0.079 \\
\text { (average-move) }\end{array}$} & 1.0 & 130.4 & 47.4 & 57.3 \\
\hline \multirow{2}{*}{$\begin{array}{c}0.13 \\
\text { (fast-move) }\end{array}$} & 2.0 & 149.3 & 20.6 & 36.9 \\
\hline
\end{tabular}

Personalized (P-) equivalent uniform dose (EUD) of the tumor relative to EUD using the standard prescription $(=100 \%)$ and estimated survival time in months. Standard (Std.) survival time was estimated by calibrating a proliferation parameter to match with the published data [19].

\section{Discussion}

In the past, many clinical trials have been conducted for the treatment of GBMs, but there has been little progress. The failures can occur at the edges of the radiation field and even in more distant locations in the brain. These patterns of failure have led to a current Phase II clinical trial to estimate the efficacy of low-dose whole brain irradiation ( $0.15 \mathrm{~Gy}$ per fraction to whole brain and 1.85 Gy per fraction to GTV for 30 fractions) to reduce the distant recurrence rate [20]. The growing understanding of tumor dynamics and proliferation, as exemplified by Swanson's model [7], provides new impetus for exploring improved treatment strategies.

Given the expense and time needed to carry out clinical trials, and given the good correspondence of the models with clinical data, we have an opportunity to provide some critical insight into some promising future directions. The purpose of our current study is to investigate the feasibility of personalizing the radiotherapy prescription scheme based on the individual tumor characteristics to increase the efficacy of tumor cell-kills using radiation. We utilized patient-specific, glioma dynamics developed and clinically validated by Swanson et al. [7]. Using their model, Rockne et al. studied the effect of the fractionation schedules, dose distribution, and radiation sensitivity parameters [12]. They concluded that hypofractionation is more effective than hyperfractionation.

Applying this model allows us to simulate the effects of tumor growth and spread of some prescription variables: treatment margins, fractional doses, and timing of boost irradiation. By applying 
dose limits on normal tissue and tumors that are currently accepted, we have limited our simulation to clinically feasible values. We limited the total prescription dose to $60 \mathrm{~Gy}$ since dose escalation beyond that point was not proven to be efficacious in improving clinical outcomes [21]. Our results indicate that individual tumor characteristics (velocity of growth, size) make a difference in the prescription scheme that leads to the minimum tumor SF (or equivalently maximum tumor EUD).

According to our study, the most significant factors in improving the treatment efficacy are the margins around $\mathrm{GTV}_{1}$ and $\mathrm{GTV}_{2}$. Although a linearly decreasing dose policy $\left(\mathrm{P}_{5}\right)$, i.e., a larger fractional dose upfront, was found to be more effective than the constant fractional dose policy $\left(\mathrm{P}_{3}\right)$, the difference from $\mathrm{P}_{3}$ was less than $3 \%$ in the tumor EUD. A sequential boost also leads to a lower SF than a concurrent boost for almost all tumor sizes and velocities; however, the EUD using the sequential boost was larger than the EUD using the concurrent boost by no more than $4 \%$. For the average- and fast-move tumors, the personalized margin in the initial phase was $6.0 \mathrm{~cm}$, which was the maximum margin used in this study. This suggests that whole brain radiotherapy in the initial phase to 20.7-38.3 Gy followed by the focal radiation to $\mathrm{GTV}_{2}$ plus $1.5 \mathrm{~cm}$ margin to $60 \mathrm{~Gy}$ (except for the fast-move, large tumors, where the optimal $\mathrm{M}_{2}$ is $3.0 \mathrm{~cm}$ ) may be beneficial for patients with averageand fast-move tumors. On the other hand, it was found to be effective to use 3.0 and $3.5 \mathrm{~cm}$ margins in the initial phase to 43.7-45.6 Gy for slow-move, small, and large tumors, respectively, and then to use a $1.5 \mathrm{~cm}$ margin in the boost phase. Wernicke et al. investigated the effect of treatment margins for GBM and concluded that treating GBM with limited margins has been achieved without compromising overall survival or changing recurrence patterns [22]. As seen in Table 3, the expected survival varies significantly depending on the tumor size and velocity. Therefore, reporting the effect of treatment margins on survival without considering the individual tumor characteristics may lead to different conclusions. We also note that increasing treatment margins without modifying the prescription dose may increase normal tissue toxicity, which can also affect survival.

The estimations of survival time (based on Swanson's empirical observation that the modeled tumor size correlates well with death [18]) indicate that personalizing the prescription scheme can potentially increase the survival time by up to 10, 16, and 22 months for slow-, average-, and fast-move tumors, respectively, even though we recognize that actual predictions of change in survival are difficult given the complex physiology involved. The largest difference in survival time between the personalized and standard prescription scheme was for the fast-move, small tumors. On the other hand, the improvement of using a personalized scheme was the least for the fast-move, large tumors.

\section{Conclusions}

This study proposes a method to personalize a prescription scheme tailored to the individual tumor characteristics, i.e., size and velocity as observed in serial MRIs. A different prescription scheme leads to the least SF (i.e., maximum EUD) for different tumor characteristics, which may explain the heterogeneous response to the same treatment among different patients. The results of this study show the potential benefit of using a PI glioma model to personalize prescription variables based on the individual tumor characteristics to improve clinical outcomes. Future work will include exploring various mathematical models and investigating the effect of the model on the personalized prescription schemes. For example, Eikenberry et al. used continuous diffusion-reaction equations to model the behavior of proliferation and migrating tumor cells, and their interactions with chemorepellents and the extracellular matrix, using stochastic transitions between migrating and proliferating glioma cells [23].

Acknowledgments: This work was supported by the Department of Radiation Oncology, University of Washington.

Author Contributions: M.K. conceived and designed the experiments; M.K. and J.K. performed the experiments and analyzed the data; J.R. and M.P. contributed reagents/materials/analysis tools; M.K. and M.P wrote the paper, J.K. and J.R. were consulted on the manuscript of the paper.

Conflicts of Interest: The authors declare no conflict of interests. 


\section{References}

1. Corso, C.; Bindra, R. Success and failures of combined modalities in glioblastoma multiforme: Old problems and new directions. Semin. Radiat. Oncol. 2016, 26, 281-298. [CrossRef] [PubMed]

2. Stupp, R.; Mason, W.P.; van den Bent, M.J.; Weller, M.; Fisher, B.; Taphoorn, M.K.; Belanger, K.; Brandes, A.A.; Marosi, C.; Bogdahn, U.; et al. Radiotherapy plus concomitant and adjuvant temozolomide for glioblastoma. N. Engl. J. Med. 2005, 352, 987-996. [CrossRef] [PubMed]

3. The Central Brain Tumor Registry of the United States. Available online: www.cbtrus.org (accessed on 10 February 2017).

4. Gunderson, L.L.; Tepper, J.E.; Bogart, J.A. Clinical Radiation Oncology, 4th ed.; Elsevier Health Sciences: Philadelphia, PA USA, 2015.

5. Gardner, W. Residual functioning following hemispherectomy for tumour and for infantile hemiplegia. Brain 1955, 78, 487-502. [CrossRef] [PubMed]

6. Ogura, K.; Mizowaki, T.; Arakawa, Y.; Ogura, M.; Sakanaka, K.; Miyamoto, S.; Hiraoka, M. Initial and cumulative recurrence patterns of glioblastoma after temozolomide-based chemoradiotherapy and salvage treatment: A retrospective cohort study in a single institution. Radiat. Oncol. 2013, 8, 97. [CrossRef] [PubMed]

7. Swanson, K.R.; Alvord, E.C.; Murray, J.D. Virtual brain tumours (gliomas) enhance the reality of medical imaging and highlight inadequacies of current therapy. Br. J. Cancer 2002, 86, 14-18. [CrossRef] [PubMed]

8. NCCN clinical practice guidelines in oncology-Central nervous system cancers. Available online: www. NCCN.org (accessed on 10 February 2017).

9. Ghose, A.; Lim, G.; Husain, S. Treatment for glioblastoma multiforme: current guidelines and Canadian practice. Curr. Oncol. 2010, 17, 52-58. [CrossRef] [PubMed]

10. Lo, S.S.; Sahgal, A.; Slotman, B.J.; Mansur, D.B.; Khouri, A.; Sloan, A.E.; Machtay, M.; Chang, E.L. What is the most appropriate clinical target volume for glioblastoma? CNS Oncol. 2013, 2, 419-425. [CrossRef] [PubMed]

11. Dhermain, F. Radiotherapy of high-grade gliomas: Current standards and new concepts, innovations in imaging and radiotherapy, and new therapeutic approaches. Chin. J. Cancer 2014, 33, 16-24. [CrossRef] [PubMed]

12. Rockne, R.; Alvord, E.C.; Rockhill, J.K.; Swanson, K.R. A mathematical model for brain tumor response to radiation therapy. J. Math. Biol. 2009, 58, 561-578. [CrossRef] [PubMed]

13. Hall, E.J.; Giaccia, A.J. Radiobiology for the Radiologist; Lippincott Williams \& Wilkins: Baltimore, MA, USA, 2006.

14. NRG Oncology. Phase III double-blind placebo-controlled trial of conventional concurrent chemoradiation and adjuvant temozolomide plus bevacizumab versus conventional concurrent chemoradiation and adjuvant temozolomide in patients with newly diagnosed glioblastoma. Available online: www.rtog.org (accessed on 10 February 2017).

15. Gay, H.; Niemierko, A. A free program for calculating EUD-based NTCP and TCP in external beam radiotherapy. Phys. Med. 2007, 23, 115-125. [CrossRef] [PubMed]

16. Niemierko, A. A generalized concept of equivalent uniform dose (EUD). Med. Phys. 1999, 26, 1101.

17. Emami, B.; Lyman, J.; Brown, A.; Cola, L.; Goitein, M.; Munzenrider, J.E.; Shank, B.; Solin, L.J.; Wesson, M. Tolerance of normal tissue to therapeutic irradiation. Int. J. Radiat. Oncol. 1991, 21, 109-122. [CrossRef]

18. Swanson, K.R.; Rostomily, R.C.; Alvord, E.C. A mathematical modeling tool for predicting survival of individual patients following resection of glioblastoma: A proof of principle. Bri. J. Cancer 2008, 98, 113-119. [CrossRef] [PubMed]

19. Stupp, R.; Taillibert, S.; Kanner, A.A.; Kesari, S.; Steinberg, D.M.; Toms, S.A.; Taylor, L.P.; Lieberman, F.; Silvani, A.; Fink, K.L.; et al. Maintenance Therapy With Tumor-Treating Fields Plus Temozolomide vs Temozolomide Alone for Glioblastoma: A Randomized Clinical Trial. JAMA 2015, 14, 23. [CrossRef] [PubMed]

20. Kwok, Y. Phase II trial of low-dose whole brain radiotherapy with concurrent temozolomide and adjuvant temozolomide in patients with newly-diagnosed glioblastoma multiforme (GCC 1224). Available online: www.ClinicalTrials.gov (accessed on 9 May 2017).

21. Badiyan, S.N.; Markovina, S.; Simpson, J.R.; Robinson, C.G.; DeWees, T.; Tran, D.D.; Linette, G.; Jalalizadeh, R.; Dacey, R.; Rich, K.M.; et al. Radiation therapy dose escalation for glioblastoma multiforme in the era of temozolomide. Int. J. Radiat. Oncol. 2014, 15, 877. [CrossRef] [PubMed] 
22. Wernicke, A.G.; Smith, A.W.; Taube, S.; Mehta, M.P. Glioblastoma: Radiation treatment margins, how small is large enough? Pract. Radiat. Oncol. 2016, 6, 298-305. [CrossRef] [PubMed]

23. Eikenberry, S.E.; Sankar, T.; Preul, M.C.; Kostelich, E.J.; Thalhauser, C.J.; Kuang, Y. Virtual glioblastoma: Growth, migration and treatment in a three-dimensional mathematical model. Cell Prolif. 2009, 42, 511-528. [CrossRef] [PubMed]

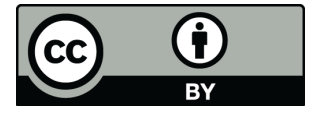

(C) 2017 by the authors. Licensee MDPI, Basel, Switzerland. This article is an open access article distributed under the terms and conditions of the Creative Commons Attribution (CC BY) license (http:/ / creativecommons.org/licenses/by/4.0/). 